\title{
EI Sistema de Clases Nominales en la lengua Saliba
}

\author{
The system of nominal classes in the Saliba Language
}

Hortensia Estrada Ramírez ${ }^{1}$

\begin{abstract}
Resumen
Una de las principales características de la lengua sáliba es su sistema de clases nominal que establece concordancia clasificatoria a lo largo de la oración. Esas clases nominales forman parte de la cosmovisión del pueblo sáliba que organiza el mundo que le rodea en dos grandes grupos: animados e inanimados. El sistema animado reúne características de género y número en tres categorías: masculino, femenino y plural o neutro. El sistema inanimado se divide en dos: singular y plural. El inanimado singular consta de veintidós clases nominales que se agrupan según su forma, función, estado o consistencia. El inanimado plural se subdivide en diez y ocho clases que son variables. Este sistema de clases parte del nombre y se ramifica en otras palabras que tiene la lengua para regir el morfema de clase que reciben. Palabras clave: Sistema de clases nominal. Morfema de clase nominal; Animado. Inanimado. Concordancia clasificatoria.
\end{abstract}

\begin{abstract}
One of the main features of the Saliba language is a distinct nominal class system that establishes a qualifying grammatical agreement along sentences. Those nominal classes belong to worldview of the Saliba people, who classify the world around them into two large groups: animate and inanimate entities. The animate system incorporates gender and number characteristics into three categories: masculine, feminine, and plural or neuter. The inanimate system splits into two categories: singular and plural. The singular inanimate family includes 22 nominal classes assorted according to their form, function, state, or consistency. The plural inanimate family is subdivided into 18 variable classes. This class system has the name as its starting point and ramifies into new words that take several class morphemes.
\end{abstract}

Keywords: Nominal class system. Nominal class morfem. Animated. Inanimated. Classificatory Concordance.

\section{Generalidades}

La lengua sáliba pertenece a la familia lingüística sáliba-piaroa. Tanto el pueblo como la lengua tienen el mismo nombre salia 'sáliva o sáliba'.

El pueblo sáliba está ubicado en Colombia y Venezuela. En Colombia son aproximadamente $2.231^{2}$ personas que habitan principalmente en el departamento de Casanare y un menor número vive en los departamentos de Vichada y Meta. En Venezuela son unas 700 personas que habitan en el Estado de Amazonas.

\footnotetext{
${ }^{1}$ Instituto Caro y Cuervo.

2 Según el censo del autodiagnóstico sociolingüístico realizado por el Programa de Protección a la Diversidad Etnolingüística, PPDE, Ministerio de Cultura en el 2008.
} 
El sáliba está en riesgo de desaparecer debido a que hay muy pocas personas que hablan este idioma. El contacto con los colonos y las compañías petroleras, los medios masivos de comunicación, los grupos religiosos y la educación escolarizada, son algunos de los principales factores que han incidido en su pérdida. Los jóvenes y los niños son monolingües en español y los adultos en su mayoría son hablantes pasivos. Solo un reducido número de la población conoce y practica la lengua en la vida cotidiana, esto sucede porque no se encuentran interlocutores para sostener conversaciones en sáliba, ya que raras veces la lengua sáliba se emplea en el hogar, en la escuela y en las reuniones comunitarias. Los pocos que la hablan, que en su mayoría son mujeres mayores entre 40 o 50 años, practican la lengua en encuentros casuales con otras mujeres sálibas ya mayores.

\section{El sistema de clases nominales y su concordancia clasificatoria}

La lengua sáliba cuenta con un sistema de clases nominales animado e inanimado que proviene del nombre y depende de él, razón por la cual los nombres animados e inanimados son los que determinan el morfema de clase que reciben las demás palabras del sintagma nominal o de la oración.

En el sistema animado se agrupan los humanos, los animales, el sol, la luna, las estrellas, los espíritus y el diablo. En el inanimado están los vegetales, los minerales y los demás elementos de la naturaleza que no tienen movimiento. El sistema de clase nominal animado e inanimado está conformado por un grupo de morfemas con sus alomorfos que se sufijan al nombre, a los determinantes del nombre, al adjetivo, al verbo y a algunos adverbios para establecer concordancia clasificatoria.

\subsection{Concordancia clasificatoria en el sintagma nominal}

La mayoría de los nombres sufijan un morfema de clase nominal que establece concordancia clasificatoria con sus determinantes, pero esta concordancia puede variar dependiendo de que ese morfema de clase sea el mismo, distinto, que solo una de las palabras lo sufije o ninguna lo sufije. Cuando un nominal animado o inanimado no sufija el morfema de clase nominal lo hace alguno de sus determinantes. La mayoría de los determinantes sufijan el morfema de clase, solo unos pocos no lo hacen. Los determinantes que sufijan el morfema de clase son: los demostrativos lejanos y cercanos, los posesivos, los interrogativos, los indefinidos, los cuantificadores y los numerales. Los determinantes que no sufijan el morfema de clase son: los cuantificadores 'todos', 'pocos (inanimado) y 'muchos' (animado). 
En el animado singular la concordancia clasificatoria es más variable e irregular que en el inanimado singular, mientras que en el inanimado plural existe un mayor número de variaciones morfémicas e irregularidades en la concordancia clasificatoria que el animado plural.

\section{Tipos de concordancia}

En esta lengua se observa que existen diferentes tipos de concordancia clasificatoria en el sintagma nominal y en la oración:

Concordancia con el mismo alomorfo: cuando los morfemas de clase del nombre y el determinante coinciden en su forma:

1. deo-be $\tilde{u}$-be gordo-CN:an.masc.sg /hombre-CN:an.masc.sg. ${ }^{3}$

'hombre gordo'

2. $p i \underline{\underline{e}} p e^{2} \underline{-e}$

Dem-CN:in.redondo y plano/casabe-CN:in.redondo y plano

'este casabe' (torta de yuca brava)

Concordancia con diferente alomorfo: cuando los morfemas de clase nominal del nombre y el determinante no coinciden en su forma debido a que la clase nominal tiene distintos alomorfos:

3. hoto-bo onea-xu

alguna-CN:an.fem.sg/joven-CN:an.fem.sg.

'una joven'

4. tuхй-dufi-mu

Num/CN:an.pl./cachorro/CN:an.pl.

'dos cachorros'

5. hi-nu botutu-pu

Dem-CN:in.pl/botuto-CN:in.pl.

'esos botutos' (instrumento musical)

\footnotetext{
${ }^{3}$ Abreviaturas: AN: animado, CN: clase nominal, CUANT: cuantificador, DAT: caso dativo, DEM: demostrativo, DUR: aspecto durativo, FEM: femenino, FOC: actante focalizado, GEN: genitivo de procedencia, IN: inanimado, INES: caso inesivo, Int: interrogativo, I.P1s: índice de primera persona singular, I.P2s: índice de segunda persona singular, I.P3SF: índice de tercera persona singular femenino, I.P3SM: índice de tercera persona singular masculino, I.P1PL: índice de primera persona plural o neutro, I.P2PL: índice de segunda persona plural o neutro, I.P3PL: índice de tercera persona plural o neutro, MASC: masculino, NEG: negación, NUM: numeral, NZDR: nominalizador, PL: plural, P1s: primera persona singular, P2s: segunda persona singular, P3SF: tercera persona singular femenino, P3sm: tercera persona singular masculino, P1 PL: primera persona plural o neutro, P2PL: segunda persona plural o neutro, P3PL: tercera persona plural o neutro, REAL: modo real, sg: singular, VIRT: modo virtual.
} 
6. $p i-\underline{-\jmath}$ kanali- $\underline{\underline{u}}$

Dem-CN:in.contenedor./tinaja-CN:in.contenedor

'esta tinaja'

Concordancia parcial: cuando una de las palabras, el nombre o el determinante, no sufija la marca de clase nominal:

7. ¿ti-di akala?

Int-CN:an.masc.sg/gallina

¿cuál gallo?

8. tie sipu-du

Cuant/estrella-CN:an.pl.

'todas las estrellas'

9. icahẽ pake-a

Cuant/totuma-CN:in.pl

'pocas totumas'

10. po-to oxe

Dem-CN:in.líquido/caño pequeño

'ese caño pequeño'

Sin concordancia: cuando no hay concordancia clasificatoria debido a que el nombre y el determinante no sufijan la marca de clase nominal:

11. tie apicha

Cuant/cerdo

'todos los cerdos'

12. hobe malibana

Cuant/pez caribe

'mucho pez caribe'

13. icahẽ ikwaa

Cuant/comida

'poca comida'

\subsection{Concordancia clasificatoria en contexto de oración}

Como ya se dijo, la concordancia clasificatoria parte del nombre y se extiende a las demás palabras de la oración que reciben el morfema de clase del nominal referido. Las palabras que sufijan este morfema son: los nombres, los determinantes del nombre, los adjetivos y los verbos. Los verbos reciben morfemas de clase nominal en oraciones focalizadas. Este tipo de predicación es bifurcada debido a que es imperiosa la presencia tanto del sujeto como del 
predicado para que la oración tengan sentido, porque el verbo se ha nominalizado perdiendo los índices actanciales que remplazaban al sujeto:

Verbos monovalentes con marcas de clase nominal: el sujeto se focaliza con el morfema de actante focalizado:

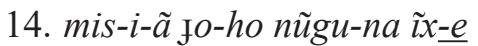

Gato-CN:an.masc.sg-FOC/Dem-CN:an.masc.sg/árbol-INES/vivir-CN:an. masc.sg

'el gato es el que vive en el árbol'

Verbos bivalentes con un morfema de clase nominal: son raros, se encuentran en oraciones focalizadas negativas, como la del ejemplo 15. que afija el morfema de clase - $x u$ 'animado femenino singular' (agente) y el morfema - $d i$ 'índice de tercera persona plural' (dativo):

15. dad-o-xa hi-xu misi-di $\tilde{o}-\tilde{a}-x u-d i-d i$

abuela-CN:an.fem.sg-FOC/Dem-CN:an.fem.sg./gato-DAT/querer- $i ?-C N$ :an. fem.sg-I.P3SM-Neg.

'La abuela odia (no quiere) a los gatos"

Verbos trivalentes con un morfema de clase nominal: el verbo afija dos índices de persona (agente y beneficiario) y el morfema de clase nominal (objeto). En este tipo de predicación, el verbo sufija el morfema de clase nominal que es el índice del objeto, mientras que el objeto externo se prepone al verbo:

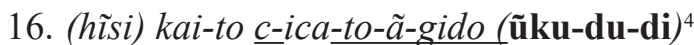

P1S/agua-CN:in.líquido/I.P3SF-dar-CN:in.líquido-Real-I.P2P1/P2S-CN:an. pl.-DAT

'Yo les doy agua a ustedes'

Cuando el morfema de clase nominal reemplaza o sustituye al índice actancial, la base verbal mantiene el morfema de aspecto durativo, que se infija entre el morfema de clase, pero se desmodaliza:

17. hi-xu-xa hi-xu maju-b-in-o

Dem-CN:an.fem.sg-FOC/Dem-CN:an.fem.sg/trabajar-CN:an.fem.sg-DUR-

CN:an.fem.sg

'ella es la que está trabajando'

Algunos verbos intransitivos que afijan la marca de clase, cuando reciben el morfema de negación, mantienen el mismo morfema de clase nominal en el animado masculino singular, pero con el animado femenino singular adicionan el morfema de clase nominal $-x u$ de esa clase:

${ }^{4}$ El agente y el beneficiario se colocan entre paréntesis porque se puede prescindir de ellos debido a que están representados con su índice en el verbo. 
18. Јo-ho gu-pe-di

Dem-CN:an.masc.sg/caminar-CN:an.masc.sg-Neg

'él no caminó'

19. hi-xu gu-pa-xu-di

Dem-CN:an.fem.sg/caminar-CN:an.masc.sg-CN:an.fem.sg-Neg 'ella no caminó'

Algunas construcciones transitivas focalizadas que reciben el morfema de negación, cambian el índice actancial agente por la marca de clase nominal:

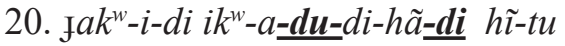
culebra-CN:an.masc.sg-DAT/comer-i?-CN:an.pl-I.P3SM-FOC-Neg/Dem-C$\mathrm{N}:$ an.pl 'ellos no comen culebra'; Lit.: culebra es lo que no comen ellos.

\subsection{Morfemas de clase nominal en el nombre}

\subsubsection{Animado}

El sistema de clases nominales del animado singular indica características de género y número. Se organiza en tres categorías: masculino, femenino y plural o neutro. Cada grupo tiene un morfema abstracto con varios alomorfos que se sufijan a nombres de humanos, animales, espíritus, el diablo, el sol, la luna y las estrellas. Esos alomorfos, aunque pertenezcan a la misma clase, no son susceptible de intercambiarse en una misma palabra porque cada palabra tiene un único morfema de clase que les inherente.

Tabla No.1: Morfemas de clase nominal animado

\begin{tabular}{|l|l|l|l|l|l|l|l|}
\hline $\begin{array}{r}\text { Animado } \\
\text { (género/úmero) }\end{array}$ & \multicolumn{7}{|c|}{ Clases nominales em el animado } \\
\hline Masculino & -e $\sim$-ẽ & -be $\sim$-ba & -pe & -me & -ho $\sim$-xo & -di $\sim$-i & \\
\hline Femenino & -o & -bo & -po & & & -xu & \\
\hline Plural o neutro & -odu & -bodu & -podu & -um & & -du $\sim$-tu & $\begin{array}{l}\text {-bai } \sim \text {-baju } \\
\sim \text {-nu }\end{array}$ \\
\hline
\end{tabular}

\subsubsection{Animado masculino singular}

CN -e $\sim$-ẽ $\sim$-be

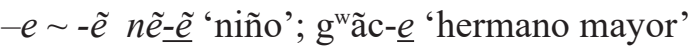

-be ũ-be 'hombre' 


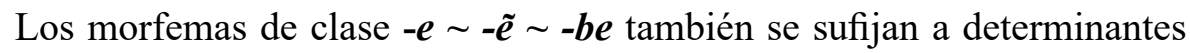
del nombre, adjetivos y verbos nominalizados. Este grupo también tiene el alomorfo - $\boldsymbol{b} \boldsymbol{a}$ que aparece en determinantes del nombre y el alomorfo -pe solo se presenta en construcciones verbales que se nominalizan.

CN - ho $\sim$-xo

-ho be-ho 'luna'; g'aigo- $\underline{h o}$ 'guahibo' (grupo étnico)

-xo ho-xo 'persona'; hĩ-xo 'gente'; ho- xo-te 'sol'

CN -di $\sim-\mathbf{i}$

-di sipo-di 'estrella'; nopõ-di 'coloradito' (cierto insecto)

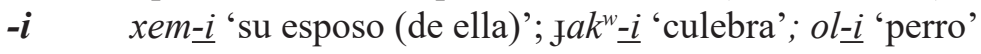

\subsubsection{Animado femenino singular}

CN -o

-o caf-O 'mi cuñada'; nopod-o 'mi madrina'

Esta grupo también tiene el alomorfo -bo que solo se presenta en indefinidos, cuantificadores, adjetivos y verbos y el alomorfo -po que aparece en construcciones verbales con tendencia a la nominalización.

$\mathrm{CN}-\boldsymbol{x u} \sim-\boldsymbol{u}$

-хи и- $u$ - 'sapo'; na-xu 'mujer'

-u cac-u 'mi hermana (genérico)'; $g^{w} a c \underline{u}$ 'mi hermana menor'

\subsubsection{Animado plural o neutro}

El animado plural también se puede llamar neutro porque no establece ninguna distinción de género. Funciona de manera similar a las dos clases anteriores.

\section{CN-bodu}

-bodu cume-bodu 'mis hermanos', cinase-bodu 'mis suegros'

Este grupo también tiene el alomorfo -odu que solo se sufija a verbos y adjetivos y el alomorfo - podu solo se sufija a verbos.

$\mathrm{CN}-d u \sim-t u$

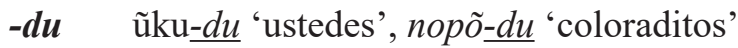

-tu cemi-tu 'familiares', nẽ-tu 'niños'

$\mathbf{C N}-\mathrm{n} \boldsymbol{u}$

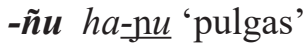

$\mathbf{C N}-\mathbf{m u}$

-mu cĩs $\underline{a-m u} \sim \operatorname{cĩs} \underline{a-m o}$ 'nietos' $\phi i-m u \sim \phi i-m o$ 'pichones' 


\section{Particularidades del sistema de clase nominal animado}

Cuando el nombre no sufija el morfema de clase del animado singular o plural, la distinción la establece alguno de sus determinantes: akala 'gallina'

21. pi-di akala

Dem-CN:an.masc.sg/gallina

'esta gallina'

22. $p i-x u$ akala

Dem-CN: an.fem.sg/gallina

'este gallo'

23. pĩ-tu akala

Dem-CN:an.pl/gallina

'estas (os) gallinas /gallos'

Nombres que sufijan un morfema de clase que distingue el número pero

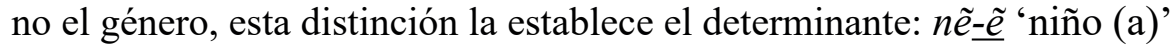

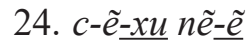

I.P1S-V-CN:an.fem.sg/niño-CN:an.masc.sg

'mi hija (fem.)'

25. $c-\tilde{l}-\underline{d i} n \tilde{e}-\underline{\tilde{e}}$

I.P1S-V-CN:an.masc.sg/niño-CN:an.masc.sg 'mi hijo (masc.)'

26. $n \tilde{e}-$ tu

niño-CN:an.pl

'niños (as)'

27. $c-\tilde{e}-\underline{-b a \mathbf{n} u} n \tilde{e}-\underline{t u}$

I.P1S-V-CN:an.pl/niño-CN:an.pl

'mis hijos (as) (neutro)'

Nombres que sufijan el morfema de clase nominal que aparentemente es $-e$ : 'masculino singular' y que no distinguen el número en el plural, la distinción de género y número la establece el determinante:

cee ‘cachicamo o armadillo' (hembra o macho)

28. $h i \underline{-x u}$ ce $\underline{-e}$

Dem-CN:an.fem.sg/cachicamo-CN:an.masc.sg

'cachicamo (hembra)'

29. Jo-ho ce-e

Dem-CN:an.masc.sg/cachicamo-CN:an.masc.sg

'cachicamo (macho)' 
30. hĩ-tu ce-e

Dem-CN:an.pl/cachicamo-CN:an.masc.sg 'cachicamos'

Algunos nombres de humanos tienen un único género, tales como: mujer, hombre y niño:

31. $\mathrm{n} a-x u$

mujer-CN:an.fem.sg

'mujer'

32. $\tilde{u}-b e$

hombre-CN:an.masc.sg

hombre'

Existen otros nombres de humanos que no identifican género, por ejemplo, 'persona', 'gente', 'indígena' que son nombres neutros y genéricos, sufijan el morfema de clase $-x o$ :

33. ho- $\underline{x o}$

persona-CN:an.neutro

'persona'

34. $h \tilde{\imath}-\underline{x o}$

gente-CN:an.neutro

'gente'

Algunos nombres de animales, debido a razones aún no identificadas, en la cultura sáliba solo son hembras o machos, por ejemplo, 'sapo' es hembra únicamente, y por consiguiente sólo sufija los morfemas de clase $-x u$ 'femenino singular' y $-t u$ 'femenino plural':

35. $u-x u$

sapo-CN:an.fem.sg

'sapo' (hembra)

36. $u-\underline{t u}$

sapo-CN:an.pl

'sapos' (plural neutro)

Otros nombres como 'sol' sufijan dos morfemas de clase nominal; uno animado y otro inanimado. El morfema de clase nominal animado $-x o$ y el morfema de clase nominal inanimado-te: corto y redondo:

37. ho-xo-te

sol-CN:an.neutro-CN:in.corto y redondo

'sol' 
Nombres de animales como 'murciélago', que en el singular sufija el morfema de clase - ga $^{5}$ que aparentemente es neutro y el morfema - en el plural:

38. $k^{w} a \tilde{x} u-g a$

murciélago-CN:an.sg $i$ ?

'murciélago' (macho)

39. $\mathrm{k}^{\mathrm{w}}$ aĩxu-

murciélago-CN:an.pl

'murciélagos (machos)'

Nominales que mantienen el morfema de clase del singular y afijan el morfema del plural o neutro:

40. $g^{w} a c-\underline{e}$

hermano-CN:an.masc.sg

'hermano mayor'

41. $g^{w} a c-e-b o d u$

hermano-CN:an.masc.sg-CN:an.pl

'hermanos mayores'

Nominales que no reciben ninguna marca de clase en el singular pero que en el plural si la reciben:

42. nana

mamá

'mamá'

43. nana-bodu

mamá-CN:an.pl

'mamás'

Nombres de animales préstamo del español que reciben un morfema de clase nominal en el plural que no determina el género pero sí el número:

44. paka-

vaca-CN:an.sg

'vaca'

45. paka-ha

vaca-CN:an.pl.

'ganado'

\subsubsection{Inanimado}

El sistema de clases del inanimado reúne las plantas, los minerales, el vestido, la vivienda, el menaje y ajuar doméstico y todos los elementos que

${ }^{5}$ Tiene la misma forma del genitivo de procedencia. 
forman parte de la vida cotidiana del pueblo sáliba. Sus morfemas de clase se identifican por características cualitativas y cuantitativas de forma, función, y estado o consistencia.

\subsubsection{Inanimado singular}

Los morfemas de clase se sufijan a los nombres, y si el nominal es el sujeto de una oración, su morfema de clase nominal rige las demás palabras de la construcción. El inanimado singular cuenta con veintidós (22) morfemas de clase nominal en total:

\section{Clasificación por la forma}

\begin{tabular}{|c|c|c|}
\hline$-p u$ & Redondo o circular & $\begin{array}{l}\text { bahi-pu 'molde para hacer tinajas' (vasija } \\
\text { de barro); obi-pu 'aro' }\end{array}$ \\
\hline$-t e$ & $\begin{array}{l}\text { Parte de un todo, corto y } \\
\text { redondo }\end{array}$ & $\begin{array}{l}\text { paxu-te 'ojo'; dulai-te 'asiento (tronco de } \\
\text { madera)' }\end{array}$ \\
\hline$-c u$ & Pequeño, duro y redondo & $\begin{array}{l}\text { ina-cu 'piedra'; cahã-cu '(mi) barbilla, } \\
\text { mentón' }\end{array}$ \\
\hline$-x u \sim-x \tilde{u}$ & Hueco y/o circular & 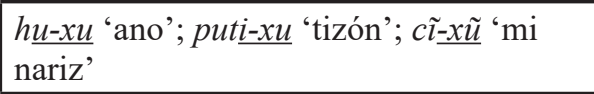 \\
\hline$-x o \sim-x$ & $\begin{array}{l}\text { Ovalado y/o cóncavo, } \\
\text { mitades }\end{array}$ & 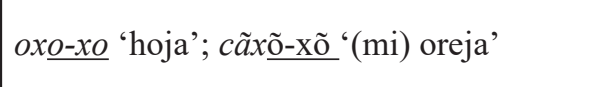 \\
\hline$-d e$ & Ancho y/o plano & $\begin{array}{l}k \tilde{a}-d e \text { 'sabana'; denai-de 'canalete'; bate- } \\
\underline{d e} \text { 'batea' (plato de madera) }\end{array}$ \\
\hline$-m o$ & $\begin{array}{l}\text { Abultado y/o abierto por un } \\
\text { extremo }\end{array}$ & $\begin{array}{l}\text { ofo-mo 'olla'; bototu-mo 'botuto' } \\
\text { (instrumento musical) }\end{array}$ \\
\hline $\begin{array}{l}-h a \sim h \tilde{a} \sim \\
-a \sim-\end{array}$ & Alargado vertical & $\begin{array}{l}\text { ece-ha 'batata'; hẽka-hã 'sebucán' } \\
\text { (exprimidor de yuca); dopes-a 'pilón' }\end{array}$ \\
\hline$-t a$ & $\begin{array}{l}\text { Planta alargada o en forma } \\
\text { de caña }\end{array}$ & $\begin{array}{l}\text { Jomai-ta 'mata de maíz'; alosai-ta 'mata } \\
\text { de arroz'; noh } a i-t a \text { 'mata de caña' }\end{array}$ \\
\hline$-k^{w} a \sim-k^{w} e$ & $\begin{array}{l}\text { Alargado y/o con picos o } \\
\text { puntas }\end{array}$ & 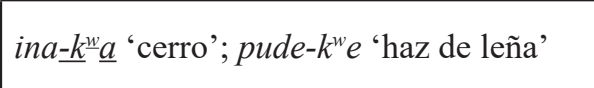 \\
\hline$-c e \sim-c \tilde{e}$ & $\begin{array}{l}\text { Alargado y/o agrupado en } \\
\text { racimos, conjuntos, etc. }\end{array}$ & 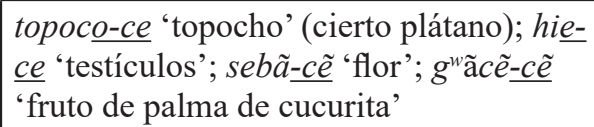 \\
\hline$-n o$ & Alargado & doko-no 'flecha'; paca-no 'badea' \\
\hline$-b o \sim-b a$ & $\begin{array}{l}\text { Filiforme, delgado y largo, } \\
\text { aplanado }\end{array}$ & $\begin{array}{l}\text { põhai-bo 'hilo'; onani-ba 'cabuya'; } \\
\text { pudu-ba 'escopeta' }\end{array}$ \\
\hline$-\boldsymbol{h} \tilde{\boldsymbol{e}}$ & $\begin{array}{l}\text { Delgado, menudo y/o } \\
\text { puntudo }\end{array}$ & 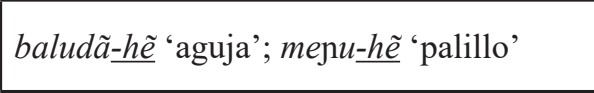 \\
\hline$-x e \sim-x \tilde{e}$ & $\begin{array}{l}\text { Cubiertas cóncavas o } \\
\text { convexas }\end{array}$ & 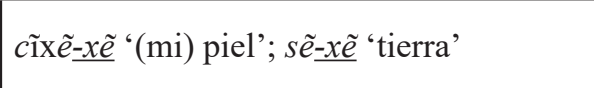 \\
\hline
\end{tabular}




\begin{tabular}{|l|l|l|}
\hline$-\mathrm{n} \boldsymbol{u}$ & Palmácea & $\begin{array}{l}\text { hõta-nu 'escoba'; gwãca-nu 'palma de } \\
\text { cucurita' }\end{array}$ \\
\hline $\boldsymbol{-} \boldsymbol{e} \sim-\mathrm{\jmath} \boldsymbol{e}$ & Redondo y plano & $\begin{array}{l}\text { pe } e^{\text {-e }} \text { 'cazabe'; teta-e 'plato'; sãp } \underline{a-e} \\
\text { 'arepa' }\end{array}$ \\
\hline
\end{tabular}

\section{Clasificación por la función}

\begin{tabular}{|l|l|l|}
\hline $\begin{array}{l}-\boldsymbol{\jmath} \boldsymbol{u} \sim-\boldsymbol{u} \sim-\boldsymbol{h u} \\
\sim-\boldsymbol{h o} \sim \boldsymbol{- o}\end{array}$ & Contener, guardar, alojar & $\begin{array}{l}\text { nügu-fu} \text { 'árbol'; kanali-u 'tinaja'; afi-hu} \\
\text { 'ahuyama'; } g^{w} e c \underline{\underline{u}-h o} \text { 'canoa' }\end{array}$ \\
\hline
\end{tabular}

\section{Clasificación por el estado o la consistencia}

\begin{tabular}{|c|c|c|}
\hline- to & Líquido & $\begin{array}{l}\text { kai-to 'agua'; leci-to 'leche'; ac } \underline{\underline{i}-t o} \text { 'caño'; } \\
\text { ok } \underline{\underline{o-t o}} \text { 'caldo' }\end{array}$ \\
\hline$-n e$ & Consistencia blanda & $\begin{array}{l}\text { cana-ne 'mi lengua'; îku-ne 'tungo' } \\
\text { (envuelto de maíz), afak } \underline{a-n e} \text { 'hayaca' } \\
\text { (tamal) }\end{array}$ \\
\hline$-\boldsymbol{h}^{\boldsymbol{w}} \sim \boldsymbol{h} \boldsymbol{o}^{6}$ & $\begin{array}{l}\text { Partículas granuladas no } \\
\text { contables }\end{array}$ & 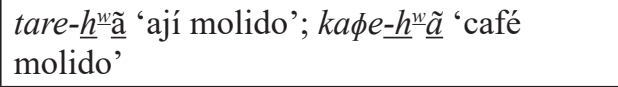 \\
\hline
\end{tabular}

\section{Características no precisadas}

\begin{tabular}{|l|l|l|}
\hline$-\boldsymbol{m a} \sim-\boldsymbol{m} \boldsymbol{e}$ & Lugar y otros no precisados & $\begin{array}{l}\text { pe-me ma:na 'camino'7; sahi-me 'conuco } \\
\text { nuevo' (campo de cultivo); sãp } \underline{a-m e} \text { 'arepa'; } \\
\text { ajata-me 'tapa de la olla' }\end{array}$ \\
\hline
\end{tabular}

En el inanimado existen clases que tienen un único morfema, tales como: -pu, -te, -e, - $k^{w} e-, c u,-x \tilde{e},-d e,-m o,-t a,-h \tilde{e},-\mathrm{n} u,-t o,-n e$ y -no, sin embargo, esto no significa que haya una sola denominación en estas clases, en ellas se pueden agrupar diferentes nombres de acuerdo con la característica que las unan. Esos morfemas se sufijan a las distintas palabras para establecer la misma concordancia clasificatoria a lo largo de la oración:

\section{6. kamisa- $k^{w} \underline{e}$ hi- $\underline{k}^{w} \underline{e}$ nõg ${ }^{w} e-n a g^{w} e l e-k^{w} \underline{e}$}

camisa-CN:in.alargado, con picos/Dem-CN: in.alargado, picos/vara-INES/

colgar-CN:in.alargado, picos

'la camisa está en la vara'

\footnotetext{
${ }^{6}$ El morfema de clase nominal -ho parece ser que es una variante que se utiliza con mayor frecuencia en Morichito, resguardo de Caño Mochuelo, municipio de Hato Corozal (Casanare).

${ }^{7}$ Algunos nombres no sufijan el morfema de clase nominal, lo sufijan sus determinantes, como en ma:na 'camino'.
} 
Algunos alomorfos son orales o nasales porque el morfema que les precede es oral o nasal, tales como $-x o \sim-x \tilde{o} ;-x u \sim x \tilde{\mathrm{u}} ;-c e \sim-c \tilde{e}$

47. hide-ce te:-ce

Int-CN: in.alargado, agrupado/estómago-CN: in.alargado, agrupado

¿¿Cuántos estómagos?'

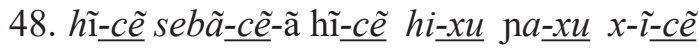

Dem-CN: in.alargado, agrupado/flor-CN: in.alargado, agrupado-FOC/Dem$\mathrm{CN}$ :in.alargado, agrupado/Dem-CN:an.fem.sg/mujer-CN: an.fem.sg/I.P3SF-

V-CN: in.alargado, agrupado

'esa flor es de aquella mujer'

Otro tipo de marcas de clase nominal que tienen más de un alomorfo son: la $\mathrm{CN}$ : $-a$ que tiene los alomorfos $\{-h a,-h a ̃,-f a\}$ y la $\mathrm{CN}$ : $-\mathrm{f} u$ que tiene los alomorfos $\{-u,-h u,-h o,-o\}$, que pueden sufijarse a las distintas clases de palabras que conforman la oración, estableciendo una concordancia clasificatoria con el alomorfo correspondiente a la clase nominal en mención:

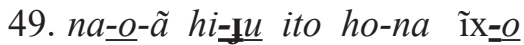

vestido-CN: in.contenedor-FOC/Dem-CN:in.contenedor/casa/dentro-INES/

estar-CN: in.contenedor

'el vestido está adentro de la casa'

50. $h i-\_a$ tesi-a suda-ha tãda-ha hĩhi-Ia sĩgwe-ha

Dem-CN:in.alargado/chinchorro-CN:in.alargado/feo-CN:in.alargado/negro-C-

$\mathrm{N}$ :in.alargado/Dem-CN:in.alargado/viejo-CN:in.alargado

'ese feo chinchorro negro está viejo'

Esta lengua clasifica la flora en cuatro grupos: árboles, matas en forma de caña, palmas y tubérculos. Cada uno de estos elementos del mundo vegetal tiene una raíz léxica que sufija diferentes morfemas de clase según el todo o la parte (hoja, flor, fruto o semilla) que se mencione.

Pero quizás por la importancia del maíz en la dieta alimenticia, esta raíz sufija muchos otros morfemas de clase nominal: „omo- $\mathrm{ca}$ 'mazorca sin coger',

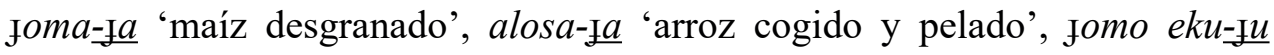
"mazorca de maíz", „omo eku-cu 'conjunto de mazorcas'.

La yuca tiene otra clasificación por ser un tubérculo de gran importancia en la dieta alimenticia. Sufija el morfema de clase nominal $-\mathbf{J} a \sim-a$ : 'alargado vertical' y distingue dos tipos de yuca: dua sẽnã 'yuca amarga o venenosa' y piki- $\underline{a} \sim$ piki-ła 'yuca dulce', con su gran cantidad de variedades.

El ají tare, de gran importancia en la cultura sáliba cuenta con otras denominaciones relacionadas con sus estados: tare $\underline{h} \underline{\underline{w}} \underline{a}$ 'ají molido', tare-te 'grano de ají', tare-to 'ají líquido'. 


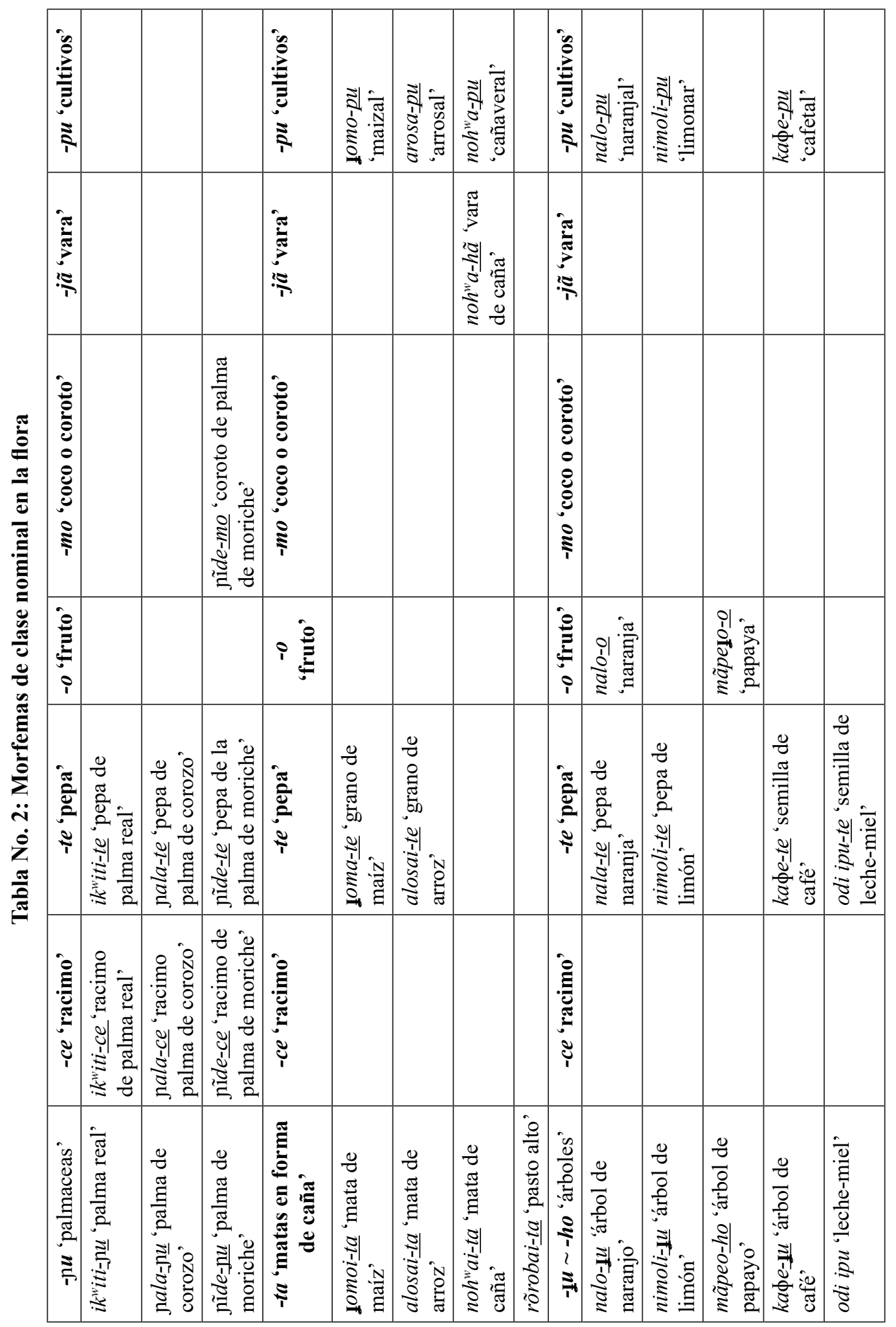


El préstamo del español kaфe 'café' también recibe las denominaciones relacionadas con sus estados: $k a \phi e \underline{-} \underline{\underline{w}} \underline{a}$ 'café en polvo' y kaфe-to 'café líquido'.

Los morfemas de clase nominal que se pueden permutar para dar características diferentes de un elemento, no son exclusivos del reino vegetal, otros elementos construidos por el hombre también pueden cambiar la marca de clase nominal:

51. oэo-pu

olla-CN:in.redonda

'olla redonda'

52. oэo-mo

olla-CN:in.hueca

'olla hueca'

53. oэo-he्e

olla-CN:in.filiforme y/o delgad

'olla estrecha'

54. sãpa-e

arepa-CN:in.redonda y plana

'arepa redonda y plana'

55. sãpa-i-me

arepa-CN: in.alargado

'arepa alargada'

Estos cambios de morfemas de clase en un mismo nominal dependen mucho del contexto de la oración y de la función o característica que interese destacar:

56. ĩku-ne-ã hi-nu оэo-pu-na ĩxa

tungo-CN:in.consistencia blanda y alargado-FOC/Dem-CN:in.pl/olla-CN:in. redondo-INES/estar

'los tungos están en la olla (redonda)'

57. pe-na hi-mo-te oృo-mo ĩxa

aquí-INES/núm-CN:in.abultado y abierto por un extremo-CN:in.parte de un todo/olla-CN:in.abultado y abierto por un extremo/estar

'aquí está una olla (abultada y abierta por un extremo)'

58. оэo-mo-a tãda-mo

olla-CN:in.abultado y abierto por un extremo-FOC/negra-CN:in.abultado y abierto por un extremo

'la olla (abultada y abierta por un extremo) es negra' 
En la oración 56. se resalta la función, es decir, la olla como algo redondo que sirve para almacenar los tungos o envueltos de maíz, mientras que en las oraciones 57. y 58., que le siguen, se destacan las características de la olla (abultada y abierta por un extremo), dando mayores detalles sobre la olla misma ya que el propósito es hablar de las características de la olla y no de su función.

\section{Duplicación de las marcas de clase nominal.}

La lengua les da un tratamiento especial a nominales como el chinchorro, la casa y el vestido, posiblemente porque son pertenencias o bienes personales que están íntimamente relacionadas con su dueño. El nominal ito 'casa', cuando se menciona como pertenencia, nasaliza la $i$ - y pierde el morfema -to, para sufijar la marca de clase $-x u$ 'hueco' que se duplica. Pero si se menciona la pertenencia de la casa para indicar distancia (lejos o cerca), se mantiene la marca de clase $-x u$ que se vuelve - $x a$ por asimilación regresiva con el morfema de clase -me 'lugar', veamos:

59. ito

casa

'casa'

60. $c-\underline{-1-x u-x u}$

I.P1S-casa-CN:in.hueco-CN:in.hueco

'mi casa'

61. c-ĩ-xa-me oto -ĩx-a

I.P1S-casa-CN:in.hueco-CN:in.lugar/lejos/I.P3S-estar-REAL 'mi casa está lejos'

62. nana c-on-o x-ẽpi-a-ha $x$-ĩ-xu-xu-na ĩx-e-o-ha mamá/I.P1S-vestido-CN:in.contenedor/I.P3SF-traer-REAL-I.P1S/I.P3S-C$\mathrm{N}$ :in.hueco-CN:in.hueco

INES/quedar-?-?-I.P1S

'mi mamá trajo mi vestido que se habían quedado en su casa (de ella)'

La duplicación de la marca de clase también se da cuando se señala la cercanía o la lejanía del objeto mencionado con relación al hablante. Por ejemplo, si el hablante está muy cerca (casi tocando el objeto), dice:

\section{3. pi-ne-ne-a hi-ne îku-ne}

Dem-CN:in.consistencia blanda-CN:in.consistencia blanda/Dem-CN:in.consistencia blanda/tungo-CN:in.consistencia blanda 'esto es tungo' (está muy cerca del hablante) 
Pero si el hablante no está tan cerca, a una distancia que le permita decir 'este', solo sufija un morfema de clase en el demostrativo cercano:

\section{4. pi-ne-a hi-ne îku-ne}

Dem-CN:in.consistencia blanda/Dem-CN:in.consistencia blanda/tungo-CN:in. consistencia blanda

'esto es tungo' (está cerca, pero menos que en la oración anterior)

En resumen, el morfema de clase nominal se duplica en los nombres 'casa', 'chinchorro' y 'vestido', que se vuelven inalienables al mencionarse como posesión y en el demostrativo cercano, cuando el objeto está muy cerca del hablante.

\subsubsection{Inanimado plural}

El inanimado plural es muy irregular, su funcionamiento depende del nombre que se pluraliza. $n u$ es el morfema de clase plural que sufija a los demostrativos cercano y lejano y que se utiliza con mayor frecuencia para indicar el plural. En las demás clases de palabras, sin embargo, no es la regla general, se han encontrado otros morfemas para pluralizar los inanimados, así:

Tabla No. 3: Morfemas de clase nominal del sistema inanimado plural

\begin{tabular}{|c|c|c|}
\hline $\begin{array}{l}\text { El plural mantiene la marca de } \\
\text { clase del singular }\end{array}$ & \multicolumn{2}{|c|}{$\begin{array}{l}\text { pan- } \underline{a} \text { 'frente / frentes' } \\
h i \mathrm{j} a-\mathrm{J} a \text { 'órgano(s) sexual(es) masculino(s)' }\end{array}$} \\
\hline $\begin{array}{l}\text { El plural pierde la marca de } \\
\text { clase del singular para adquirir el } \\
\text { morfema - }\end{array}$ & \multirow{3}{*}{ 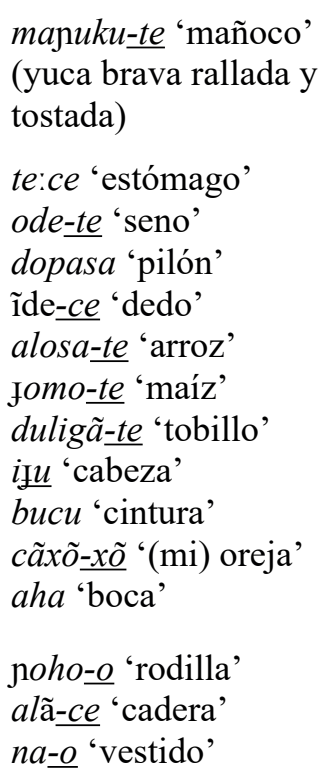 } & majuku_ 'mañocos' \\
\hline $\begin{array}{l}\text { Morfema -a, -ha, -fa, -na, -ana, } \\
\text {-bana } \pm^{8} \text { morfema de clase del } \\
\text { singular }\end{array}$ & & 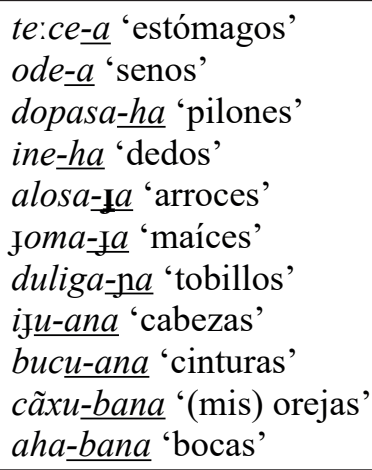 \\
\hline $\begin{array}{l}\text { Morfema -nu, - } \boldsymbol{b a j} \boldsymbol{u} \pm \text { morfema } \\
\text { de clase del singular }\end{array}$ & & $\begin{array}{l}\text { noha-n } u \text { 'rodillas' } \\
\text { ala- } \underline{\mathrm{n} u} \text { 'caderas' } \\
\text { na-baju} u \text { 'vestidos' }\end{array}$ \\
\hline
\end{tabular}

${ }^{8}$ El signo \pm significa que puede o no estar el morfema de clase singular. 


\begin{tabular}{|c|c|c|}
\hline El plural recibe el morfema $-\mathfrak{J} \boldsymbol{u}$ & $\begin{array}{l}\text { roko 'pisillo' (carne } \\
\text { en polvo) } \\
\text { sac-u 'conuco' } \\
\text { (campo de cultivo) }\end{array}$ & $\begin{array}{l}\left.\operatorname{rok}^{w} a-\right\rfloor \underline{u} \text { 'pisillos' } \\
\text { sac- } \underline{\underline{u-\jmath} u} \text { 'conucos' }\end{array}$ \\
\hline $\begin{array}{l}\text { El plural recibe el morfema }-\boldsymbol{x u} \\
\text { (a) }\end{array}$ & ito 'casa' & ito-xua 'casas' \\
\hline $\begin{array}{l}\text { Morfema -pu, }-\boldsymbol{p u a} \pm \pm \text { morfema de } \\
\text { clase del singular }\end{array}$ & 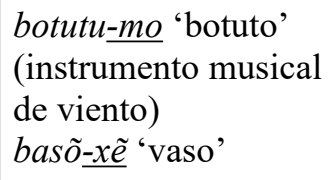 & $\begin{array}{l}\text { botutu-pu 'botutos' } \\
\text { basu-pu-a 'vasos' }\end{array}$ \\
\hline $\begin{array}{l}\text { Morfema }-\boldsymbol{d e} \pm \text { morfema de clase } \\
\text { del singular }\end{array}$ & $\begin{array}{l}\text { lepe-ce 'camaza' } \\
\text { (recipiente hecho del } \\
\text { fruto del totumo) }\end{array}$ & lepe-de 'camazas' \\
\hline 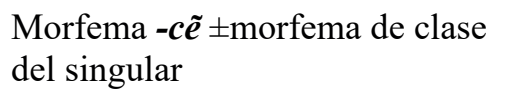 & pãhĩã-ı $a$ 'hueso' & $\begin{array}{l}\text { pãhĩa-cé } \tilde{e}^{6} \text { 'osamenta, } \\
\text { esqueletos' }\end{array}$ \\
\hline $\begin{array}{l}\text { Morfema -pe(a), -pa } \pm \text { morfema } \\
\text { de clase del singular }\end{array}$ & $\begin{array}{l}\text { teta-e 'plato' } \\
\text { tãda-e 'noche' } \\
\text { pahĩxe }-b a \text { 'párpado' } \\
h^{w} \text { ãhã 'bajo' (terreno } \\
\text { inundadizo) }\end{array}$ & $\begin{array}{l}\text { teta-pe 'platos' } \\
\text { tãda-pe-a 'noches' } \\
\text { pahĩxe-pa 'párpados' } \\
h^{w} \tilde{h} h \tilde{a}-\underline{p a} \text { 'bajos' }\end{array}$ \\
\hline El plural modifica la base léxica & ĩdece 'dedo' & ineha 'dedos' \\
\hline
\end{tabular}

El sistema de clase nominal inanimado plural es complejo. Posiblemente constituya un subsistema del sistema nominal inanimado. Algunos términos mantienen el morfema de clase nominal inanimado singular y añaden el morfema del plural. A diferencia del sistema de clase nominal inanimado singular que algunas veces puede intercambiar de marca de clases, el plural es más estable, afija un único morfema de clase que no es intercambiable.

\subsection{Morfemas de clase nominal que indican características complementarias}

Los nominales animados e inanimados pueden recibir morfemas complementarios, aparentemente de clase nominal, sin que pierdan la marca de clase que les es inherente o habitual. Dos de estos morfemas también son los derivativos: -be 'aumentativo' y hẽ- 'diminutivo' que adquieren otros significados; -be 'grande y gordo' en el animado y 'grande y grueso'; he्'pequeño y delgado' en el animado y el inanimado. 
Este subsistema de clases cumple funciones adjetivales, destaca cualidades adicionales de un nominal, estas cualidades no le son inherentes y tampoco están contempladas en el sistema de clases nominal animado e inanimado. Pueden tener la misma forma pero el significado es distinto, por ejemplo, en el sistema general de clases nominales - ce es un morfema inanimado que significa 'elemento alargado y agrupado', en este subsistema también se sufija a inanimados y significa 'feo y remendado'.

A continuación se relacionan los morfemas encontrados (no corresponden a una búsqueda exhaustiva de esta clase de morfemas):

Tabla No. 4: Morfemas de clase complementarios

\begin{tabular}{|l|l|l|}
\hline CN & \multicolumn{1}{|c|}{ ANIMADO } & \multicolumn{1}{c|}{ INANIMADO } \\
\hline$-m o$ & Joven y gordo & Enterizo \\
\hline$-j \tilde{e}$ & Pequeño y delgado & Pequeño o delgado \\
\hline$-b e$ & Grande y gordo & Grande y grueso \\
\hline$-k w a$ & Alto y delgado & \\
\hline$-d e$ & & Grande \\
\hline$-c e$ & & Feo y remendado \\
\hline$-t e$ & & Corto \\
\hline$-h a$ & & En buen estado \\
\hline$-b a$ & & Aplanado o plancheto \\
\hline
\end{tabular}

\section{5. u-be}

hombre-CN:an.masc.sg

'hombre'

66. ũ-be-mo

hombre-CN:an.masc.sg-CN:joven y gordo

'hombre (joven y gordo)'

67. ũ-be-he्e

hombre-CN:an.masc.sg-CN:delgado

'hombrecito (hombre delgado y pequeño)'

68. ũ-be-be

hombre-CN:an.masc.sg-CN:grande y gordo

'hombrón (grande y gordo)'

69. ũ-be $-k^{w} \underline{a}$

hombre-CN:an.masc.sg-CN:alto y delgado

'hombre (alto y delgado)' 
70. omota-te

blusa-CN:in.corto y redondo

'blusa'

71. omata-te-mo

blusa-CN:in.corto y redondo-CN:enterizo

'blusa enteriza'

72. omata-te-ce

blusa-CN:in.corto y redondo-CN: feo y remendado

'blusa fea y remendada'

73.omata-te-de

blusa-CN:in.corto y redondo-CN: grande

'blusa grande'

74. omata-te-he्e

blusa-CN:in.corto y redondo-CN: pequeño

'blusa pequeña'

Los préstamos del español se diferencian porque pierden la marca de clase que habitualmente afijan para adquirir los morfemas de clase con función adjetival:

75. kamisa-hẽ

camisa-CN:pequeño

'camisa pequeña'

76. kamisa-te

camisa-CN:corto

'camisa corta'

77. kamisa-mo

camisa-CN: enterizo

'camisa enteriza'

78. kamisa-ce

camisa-CN:feo y remendado

camisa fea y remendada'

\section{Otras funciones de los morfemas de clase nominal}

Se encontró que el morfema de caso del genitivo de procedencia incorpora la marca de clase del nominal animado o inanimado en mención, así: - ge 'caso genitivo animado masculino singular'; -go 'caso genitivo animado femenino singular'; -godu 'caso genitivo animado plural o neutro; -ga 'caso inanimado', por ejemplo: 
79. $s u k^{w} a-g e s \tilde{1 g}^{w}-e$

Pueblo-GEN/viejo-CN:an.masc.sg

'el viejo del pueblo'

80.suk $k^{w}$-go sĩg-o

pueblo-GEN/viejo-CN:an.fem.sg

'la vieja del pueblo'

81.pahĩ-tu-ja hĩ-tu do'do-godu sudo-du

pescado-CN:an.pl-FOC/Dem-CN:an.pl/río-GEN/feo-CN:an.pl

'los pescados de río (Meta) son feos'

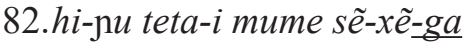

Dem-CN:an.pl/nube-CN:an.pl/encima/tierra-CN:in.cóncavo o convexo-GEN 'esas nubes del cielo'.

\subsection{Morfemas de clase en determinantes del nombre y adjetivos}

\subsubsection{Animado}

Los morfemas de clase nominal de los determinantes del nombre y de los adjetivos coinciden algunas veces con lo que se sufijan al nombre. Cada determinante del nombre y adjetivo sufija un morfema de clase que depende del nominal en mención:

Masculino singular

\begin{tabular}{|c|c|}
\hline$-e$ & sigg $\underline{-e}$ 'viejo'; lig-e 'arrugado' \\
\hline$-b e$ & $\begin{array}{l}\text { hoto-be-na 'algunos'; hoto-be 'uno'; deo-be 'gordo'; ico-ba-hẽ 'pe- } \\
\text { queño'; sôko-be 'bajo (de estatura)' }\end{array}$ \\
\hline$-h o$ & Io-ho 'ese, aquel'; -o- $\underline{h o}$ 'de él' \\
\hline$-d i$ & $\begin{array}{l}e k u-d i \text { 'soltero'; } p i-d i \text { 'este'(masc.); } c \tilde{i} \underline{-d i} \text { 'mi (masc.)'; ho-di 'otro'; ti- } \\
-\underline{-d i} \text { '¿cuál?'; go-di 'grande'; bãteco-di 'me paseo'; bo-di 'delgado'; } \phi \underline{i-d i} \\
\text { 'pichón' }\end{array}$ \\
\hline$-m e$ & hai-me 'joven (masc.)' \\
\hline
\end{tabular}

Femenino singular

\begin{tabular}{|c|c|}
\hline$-O$ & sigg-O 'vieja'; lĩg-O 'arrugada' \\
\hline$-b o$ & $\begin{array}{l}\text { hoto-bo-bena 'alguno'; hoto-bo 'uno'; deo-bo 'gorda'; ico-bo-hẽ 'pe- } \\
\text { queña'; sõko-bo 'baja (de estatura)' }\end{array}$ \\
\hline$-x u$ & 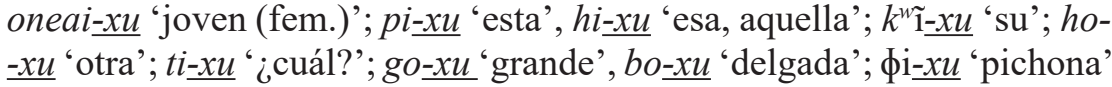 \\
\hline
\end{tabular}


Animado plural o neutro

\begin{tabular}{|c|c|}
\hline$-o d u$ & sĩg-odu 'viejos, viejas'; lĩg-odu 'arrugados, arrugadas' \\
\hline -bodu & $\begin{array}{l}\text { ico-bodu 'pequeños, pequeñas', deo-bodu 'gordos, gordas'; sõko- } \\
\underline{\text { bodu 'bajos, bajas (de estatura)' }}\end{array}$ \\
\hline$-d u$ & hĩdu-du '¿cuántos?'; hõko-du 'varios'; tuxũ $\underline{-d u}$ 'dos'; sudo-du 'feos' \\
\hline$-t u$ & $\begin{array}{l}\text { hõ-tu 'otros'; pĩ-tu 'estos (as)', hĩ } \underline{-t u} \text { 'esos, aquellos (as)'; ti-tu } \\
\text { ‘cuáles?'; go-tu 'grandes' }\end{array}$ \\
\hline$-\mathrm{nu}$ & $\begin{array}{l}e k a-\mathrm{n} \underline{u} \text { 'solteros, solteras'; bo-a-n } \underline{u} \text { 'delgados, delgadas'; tãda-n } \underline{\underline{u}} \\
\text { 'negros' }\end{array}$ \\
\hline$-m u$ & hai-mu 'jóvenes (masc.)'; $\phi \mathbf{i}-m u$ 'pichones, pichonas' \\
\hline
\end{tabular}

\subsubsection{Inanimado}

Funciona igual que el sistema animado, está regido por el nombre, el morfema de clase que sufijan los determinantes del nombre y los adjetivos dependen del nominal en mencione. Los morfemas de clase de este grupo de palabras es similar a los que a aparecen en el nombre:

83. bagĩga-k $\underline{k}^{w} \underline{e-a}$ arosa- saku_ hĩ-tu tuxũ-du be-ho pukwa-na tie bẽdega--a

cuatro-CN:in.alargado-FOC/arroz-CN:in.pl/saco-CN:in.pl/Dem-CN:an.pl/dos-

$\mathrm{CN}$ :an.pl/luna-CN:an.masc.sg/último-INES/todos/vender-I.P3SM-REAL

'En los dos últimos meses se han vendido cuatro bultos de arroz'

Lit.: se vendieron cuatro bultos de arroz en las últimas dos lunas.

84. g"ĩdi hõta-n $u g^{w} i-n u-a$ adegatea -ĩx-a

muchas/escoba-CN:in.palmacea/grandes-CN:in.palmaceas-FOC/tupidas/-I.

P3-están-REAL

'hay muchas escobas grandes y tupidas'

85. hĩda-ne $i k u=h i-n u$

INT-CN:in.consistencia blanda/tungo-CN:in.pl/Dem-CN:in.pl

‘¿cuántos tungos son?'

Es frecuente que en el nombre mantenga o pierda la marca de clase del singular en el plural.

Algunos adjetivos animados tienen un único género, tales como: joven (masculino) y joven (femenino):

86. hai-me

joven-CN:an.masc.sg

'joven (masc.)' 
87. one-ai-xu

joven-Ndzr-CN:an.fem.sg

‘joven' (fem.)

\subsection{Morfemas de clase nominal en el verbo}

El verbo establece concordancia de clase nominal con el nombre al sufijar el morfema de clase que reemplaza el índice actancial del agente, el objeto y el beneficiario. Los morfemas de clase que puede sufijar el verbo son:

\subsubsection{Animado}

Masculino singular

\begin{tabular}{|c|c|}
\hline$-e$ & ô- $\underline{\tilde{e}}^{\prime}$ el que quiere'; om-e 'el que va'; \\
\hline$-b e$ & manu-be 'el que trabaja'; kele-be 'el que hace' \\
\hline$-p e$ & $\begin{array}{l}\text { na-pe 'el que regaña'; re-pe 'el que se acuesta'; rĩ-pe 'el que canta'; to- } \underline{p-i-e} \text { e } \\
\text { 'el que se asusta' }\end{array}$ \\
\hline$-d i$ & $d u \tilde{a}-d i$ 'le duele (a él)' \\
\hline
\end{tabular}

Femenino singular

\begin{tabular}{|c|c|}
\hline-0 & 1̃x- $\underline{O}$ 'la que está'; $i k \underline{-O}$ 'la que come' \\
\hline$-b o$ & manu-bo 'la que trabaja'; kele-bo 'la que hace' \\
\hline$-p o$ & 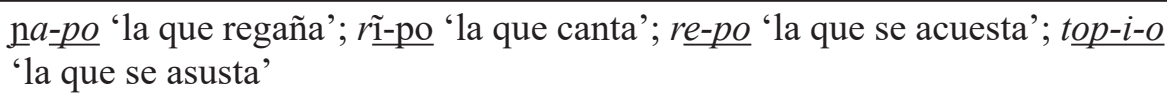 \\
\hline$-x u$ & $\begin{array}{l}\text { gudo-xu 'me voy', bãteco-xu 'me paseo', õõ-xu-didi 'ella (fem.) no lo quiere } \\
\text { a él (masc.)' }\end{array}$ \\
\hline
\end{tabular}

Animado plural o neutro

\begin{tabular}{|c|c|}
\hline$-d u$ & $i b i \underline{-d u}$ 'los, las que se bañan', sixo-du 'los, las que cosen' \\
\hline$-o d u$ & $\begin{array}{l}\text { om-odu 'los, las que vienen'; } e \underline{t-o d u} \text { 'los, las que reciben'; } a-o d u \\
\text { que duermen'; } a \underline{\text {-odu }} \text { 'los, las } \\
\text { 'los, las que tejen' }\end{array}$ \\
\hline -bodu & manu-bodu 'los, las que trabajan', kele-bodu 'los, las que hacen' \\
\hline -podu & 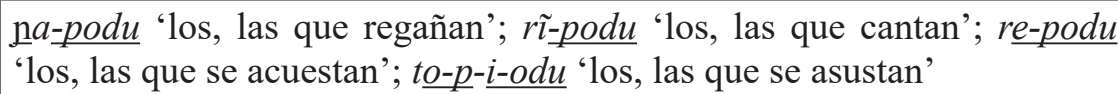 \\
\hline
\end{tabular}

\subsubsection{Inanimado}

En algunas construcciones transitivas trivalentes, el verbo puede sufijar el morfema de clase nominal del objeto en mención, que su el índice o 
representación en el verbo. Los morfemas de clase nominal inanimado son los mismos que se sufijan al nombre.

La estructura silábica del verbo cumple un papel fundamental en el tipo de morfema de clase nominal que este sufija:

- Verbos que comienzan por vocal cuya estructura silábica es: VC-, -VCVC-, -VCV-prefijan el índice de persona, pero cuando este índice se cambia por la marca de clase, sufijan el morfema de clase: $-e$ 'animado masculino singular', - $o$ 'animado femenino singular', -odu 'animado plural o neutro':

Tabla No. 5: Verbos que reciben $\mathrm{CN}$ : $-o,-e,-o d u$

\begin{tabular}{|c|c|c|c|}
\hline IND.ACT. & CN.MASc.Sg. & CN.FEM.Sg. & CN.PL. \\
\hline $\begin{array}{l}\text { c-õg }{ }^{\mathrm{w}}-\mathrm{a} \\
\text { 'yo bebo' }\end{array}$ & $\begin{array}{l}\text { õgw-e } \\
\text { 'el que bebe' }\end{array}$ & $\begin{array}{l}\text { õg-o } \\
\text { 'la que bebe' }\end{array}$ & $\begin{array}{l}\text { õg-odu } \\
\text { 'los, las que beben' }\end{array}$ \\
\hline $\begin{array}{l}\underline{\mathrm{k}}^{\underline{\mathrm{w}}}-\mathrm{ic}-\mathrm{a} \\
' \mathrm{Ud} \cdot \mathrm{da} '\end{array}$ & $\begin{array}{l}\text { ich-e } \\
\text { 'el que da' }\end{array}$ & $\begin{array}{l}\text { ic-o } \\
\text { 'la que da' }\end{array}$ & $\begin{array}{l}\text { ic-odu } \\
\text { 'los, las que dan' }\end{array}$ \\
\hline $\begin{array}{l}\underline{x}-o m-a \\
\text { 'ella va' }\end{array}$ & $\begin{array}{l}\text { om-e } \\
\text { 'el que va' }\end{array}$ & om-o & $\begin{array}{l}\text { om-odu } \\
\text { 'los, las que van' }\end{array}$ \\
\hline
\end{tabular}

- Verbos que comienzan por consonante y su estructura silábica es: V-, CVCV- y VCV-. sufijan el índice de persona, pero cuando se cambia por la marca de clase, ésta se sufija al verbo. El morfema clase nominal es: - $b e$ 'animado masculino singular', $-b o$ 'animado femenino singular', -bodu 'animado plural o neutro':

Tabla No. 6: Verbos que reciben $\mathrm{CN}$ : $-b e,-b o,-b o d u$

\begin{tabular}{|l|l|l|l|}
\hline \multicolumn{1}{|c|}{ IND.ACT. } & \multicolumn{1}{c|}{ CN.MAsc.SG. } & \multicolumn{1}{c|}{ CN.FEM.SG. } & \multicolumn{1}{c|}{ CN.PL. } \\
\hline $\begin{array}{l}\text { maju-x-a } \\
\text { 'ella trabaja' }\end{array}$ & $\begin{array}{l}\text { manu-be } \\
\text { 'el que trabaja' }\end{array}$ & $\begin{array}{l}\text { manu-bo } \\
\text { 'la que trabaja' }\end{array}$ & $\begin{array}{l}\text { maju-bodu } \\
\text { 'los, las que trabajan' }\end{array}$ \\
\hline $\begin{array}{l}\text { e-c-a } \\
\text { 'yo grito' }\end{array}$ & $\begin{array}{l}\text { e-be } \\
\text { 'el que grita' }\end{array}$ & $\begin{array}{l}\text { e-bo } \\
\text { 'la que grita' }\end{array}$ & $\begin{array}{l}\text { e-bodu } \\
\text { 'los, las que_gritan' }\end{array}$ \\
\hline $\begin{array}{l}\text { bule--a } \\
\text { 'él mueve' }\end{array}$ & $\begin{array}{l}\text { bule-be } \\
\text { 'el que mueve' }\end{array}$ & $\begin{array}{l}\text { bule-bo } \\
\text { 'la que mueve' }\end{array}$ & $\begin{array}{l}\text { bule-bodu } \\
\text { 'los, las que mueven' }\end{array}$ \\
\hline
\end{tabular}

- Verbos que comienzan por consonante y que su estructura silábica es: CV-, CVV-, CVVCV-, CVCVCV- sufijan el índice de persona, pero cuando este índice se cambia por la marca de clase, sufijan el morfema de clase: - $p$ e 'animado masculino singular', - $p o$ 'animado femenino singular', -podu 'animado plural o neutro': 
Tabla No. 7: Verbos que reciben CN: -pe, -po, -podu

\begin{tabular}{|c|c|c|c|}
\hline IND.ACT. & CN.Masc.Sg. & CN.Fem.Sg. & CN.PI. \\
\hline $\begin{array}{l}\text { nõ } \underline{-k^{\underline{w}}-a} \\
\text { 'Ud. Pela' }\end{array}$ & jõ-pe & $\begin{array}{l}\text { nõ-po } \\
\text { 'la que pela' }\end{array}$ & $\begin{array}{l}\text { nõ-podu } \\
\text { 'los, las que pelan' }\end{array}$ \\
\hline $\begin{array}{l}\mathrm{g}^{\mathrm{w}} \mathrm{i}-\mathrm{d}-\mathrm{a}-\mathrm{ad}-\mathrm{a} \\
\text { 'yo sirvo' }\end{array}$ & $\begin{array}{l}g^{\mathrm{w}} \mathrm{i}-\mathrm{p}-\mathrm{a}-\mathrm{ad}-\underline{e}^{9} \\
\text { 'el que sirve', }\end{array}$ & $\begin{array}{l}g^{\mathrm{w}} \mathrm{i}-\mathrm{p}-\mathrm{a}-\mathrm{ad}-\underline{\mathrm{o}} \\
\text { 'la que sirve', }\end{array}$ & $\begin{array}{l}\mathrm{g}^{\mathrm{w}} \mathrm{i}-\mathrm{p}-\mathrm{a}-\mathrm{ad}-\underline{\mathrm{odu}} \\
\text { 'los, las que sirven' }\end{array}$ \\
\hline $\begin{array}{l}\text { chẽbada-ha } \\
\text { 'ellos cazan' }\end{array}$ & $\begin{array}{l}\text { chẽbada-pe } \\
\text { 'el que caza' }\end{array}$ & $\begin{array}{l}\text { chẽbada-po } \\
\text { 'la que caza', }\end{array}$ & $\begin{array}{l}\text { chẽbada-podu } \\
\text { 'los, las que cazan' }\end{array}$ \\
\hline
\end{tabular}

Algunas construcciones reflexivas (afirmaciones y preguntas) reciben dos morfemas; el índice de persona y/o la marca de clase. Por lo general, son focalizadas:

88. hĩsi-ca hĩsi hĩdo $\underline{\mathbf{c}-o m-o-\underline{x u}}$

P1S-FOC/P1S/¿?/I.P1S-ir-?-CN:an.fem.sg

'yo soy la que me voy (fem.)'

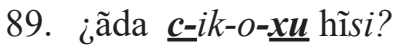

Int/I.P1S-comer-?-CN:an.fem.sg/P1S

¿qué voy a comer (fem.)?'

90. ¿tenaha $\underline{c-i b i-o-x \boldsymbol{u}}$ hĩsi?

Int/I.P1S-bañar-?-CN:an.fem.sg/P1S

¿A dónde me voy a bañar (fem.)?

Como el objeto es inanimado, su índice se representa en el verbo con la respectiva marca de clase nominal. La aparición del objeto en verbo es poco frecuente, por lo general se encuentran en respuestas a preguntas:

91. Preg:¿Quién se comió el cazabe?

Rta: $\quad c-i k^{w}-a \underline{-e}$

I.P1S-comer-REAL-CN: in.planos y redondos

'yo me lo comí (redondo y plano)'

92. Preg: ¿Quién trae el agua?

Rta: $\quad c \tilde{e}-p-o-\underline{t o}$

I.P1S-traer-?-CN:in.líquido

'yo la traigo (líquido)'

En verbos trivalentes en los que el agente y el beneficiario se representan con índices de personas y el objeto con el morfema de clase nominal, el orden de

\footnotetext{
${ }^{9}$ Nótese que el morfema de clase nominal -pe se vuelve discontinuo porque la -p- se inserta entre la raíz verbal y la -e se sufija a la misma.
} 
aparición de estos índices es: agente, objeto y beneficiario. El objeto participante externo se coloca antes del verbo:

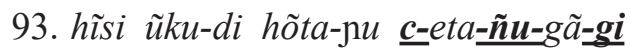

P1S-P2S-DAT/escoba-CN:in.palmácea/I.P1S-recibir-CN:in.palmacea-VIRT-I.

$\mathrm{P} 2 \mathrm{~S}$

'yo le recibo la escoba a usted'

Pero cuando el objeto no se representa en el verbo mediante la marca de clase nominal, el objeto participante externo se coloca después del verbo:

94. hĩsi üku-di c-eta-gã-gi hõta-nu

P1S/P2S-DAT/I.P1S-recibir-VIRT-I.P2S/escoba-CN:in.palmacea

'yo le recibo la escoba a usted'

\section{Nominalización del verbo}

El verbo se puede nominalizar de dos maneras: al sufijar el morfema nominalizador - $a i$ - y el morfema de clase del nominal animado - $d i$ 'masculino singular'; $-x u$ 'femenino singular $\mathrm{y}-d u$ 'masculino o femenino plural':

Tabla No. 8: Primera forma de nominalización del verbo

\begin{tabular}{|c|c|c|c|}
\hline VERBO & MASCULINO & FEMENINO & Plural o Neutro \\
\hline $\begin{array}{l}\text { koigaha } \\
\text { 'lavar' }\end{array}$ & $\begin{array}{l}\text { koig-ai-di } \\
\text { 'lavandero' }\end{array}$ & $\begin{array}{l}\text { koig-ai-xu } \\
\text { 'lavandera' }\end{array}$ & $\begin{array}{l}\text { koig-ai-du } \\
\text { 'lavanderos(as)' }\end{array}$ \\
\hline $\begin{array}{l}\text { õg }{ }^{w} a h a \\
\text { 'beber' }\end{array}$ & $\begin{array}{l}\tilde{o} g^{w} \boldsymbol{i}-a \boldsymbol{i}-\boldsymbol{d i} \\
\text { 'bebedor' }\end{array}$ & $\begin{array}{l}\tilde{o} g^{w} i-a i-x \boldsymbol{u} \\
\text { 'bebedora' }\end{array}$ & $\begin{array}{l}\tilde{\boldsymbol{o}} \boldsymbol{g}^{w} \boldsymbol{i} \text {-ai } \boldsymbol{i}-\boldsymbol{d} \boldsymbol{u} \\
\text { 'bebedores (as)' }\end{array}$ \\
\hline $\begin{array}{l}\text { heaha } \\
\text { 'correr' }\end{array}$ & $\begin{array}{l}\text { hebi-ai-di } \\
\text { 'corredor' }\end{array}$ & $\begin{array}{l}\text { hebi-ai-xu } \\
\text { 'corredora' }\end{array}$ & $\begin{array}{l}\text { hebi-ai-du } \\
\text { 'corredores (as)' }\end{array}$ \\
\hline $\begin{array}{l}\text { noaha } \\
\text { 'tejer' }\end{array}$ & $\begin{array}{l}\text { nopi-ai-di } \\
\text { 'tejedor' }\end{array}$ & $\begin{array}{l}\text { nopi-ai-xu } \\
\text { 'tejedora' }\end{array}$ & $\begin{array}{l}\text { nopi-ai-du } \\
\text { 'tejedores (as)' }\end{array}$ \\
\hline
\end{tabular}

Otros verbos se nominalizan con el morfema de clase del nominal animado en mención, no reciben ningún morfema derivativo nominalizador, basta con el morfema de clase nominal para nominalizarse:

Tabla No. 9: Segunda forma de nominalización del verbo

\begin{tabular}{|l|l|l|l|}
\hline VERBo & MaSCULINO & FEMENINO & Plural o NeUtro \\
\hline $\begin{array}{l}\text { aaha } \\
\text { 'dormir' }\end{array}$ & $\begin{array}{l}\text { a-e } \\
\text { 'el que duerme' }\end{array}$ & $\begin{array}{l}\text { a-o } \\
\text { 'la que duerme' }\end{array}$ & $\begin{array}{l}\boldsymbol{a} \text {-odu } \\
\text { 'los, las que duermen' }\end{array}$ \\
\hline $\begin{array}{l}\text { huhuaha } \\
\text { 'rezar' }\end{array}$ & $\begin{array}{l}\text { huhu-be } \\
\text { 'el que reza' }\end{array}$ & $\begin{array}{l}\text { huhu-bo } \\
\text { 'la que reza' }\end{array}$ & $\begin{array}{l}\text { huhu-bodu } \\
\text { 'los, las que rezan' }\end{array}$ \\
\hline
\end{tabular}




\begin{tabular}{|l|l|l|l|}
\hline $\begin{array}{l}\text { seaha } \\
\text { 'llorar' }\end{array}$ & $\begin{array}{l}\text { se-pe } \\
\text { 'el que llora' }\end{array}$ & $\begin{array}{l}\text { se-po } \\
\text { 'la que llora' }\end{array}$ & $\begin{array}{l}\text { se-podu } \\
\text { 'los, las que lloran)' }\end{array}$ \\
\hline $\begin{array}{l}\text { teagaha } \\
\text { 'hablar' }\end{array}$ & $\begin{array}{l}\text { te-p-ag-e } \\
\text { 'el que habla' }\end{array}$ & $\begin{array}{l}\text { te-p-ag-eo } \\
\text { 'la que habla'5 }\end{array}$ & $\begin{array}{l}\text { te-p-ag-odu } \\
\text { 'los, las que hablan' }\end{array}$ \\
\hline
\end{tabular}

En las construcciones negativas y reflexivas son más frecuentes los morfemas de clase nominal: $-d i,-x u,-d u$, mientras que en las construcciones bifurcadas en las que se focaliza el sujeto o el predicado son más usuales los verbos nominalizados con las marcas de clase nominal: $-e,-o$, -odu o sus respectivos alomorfos.

\section{Conclusiones}

El sistema de clases nominales en la lengua sáliba es finito, establece concordancias clasificatorias con casi todas las palabras de la construcción (nombres, determinantes del nombre, adjetivos y verbos). Esta concordancia parte del nombre y se extiende a las demás palabras que conforman una oración. Quizás por esta misma razón las clases nominales aunque muchas veces presentan variaciones morfémicas en una misma clase, siempre son fijas y determinadas para cada palabra, es decir, que no se pueden permutar por otro morfema de la misma clase porque cambia el sentido de la palabra o pierde su significado y los sálibas que hablan bien la lengua no se equivocan al mencionar los morfemas de clase nominal que pueden recibir las distintas palabras al mencionarlas solas o en contexto de oración. En contexto de oración las oraciones focalizadas presentan con mayor frecuencia esta concordancia de clase, en especial, las oraciones existenciales y estativas en las que el verbo recibe un morfema de clase nominal. Los verbos se pueden nominalizar de dos maneras; con un morfema nominalizador más un morfema de clase nominal o solo con un morfema de clase nominal, lo que significa que los morfemas de clase nominal cumplen un papel fundamental en el cambio de categoría gramatical de las palabras de la lengua sáliba. Existe también, un subsistema de clases con funciones adjetivales, que se ha estudiado poco, pero que por lo que se observa, señalan cualidades adicionales en el nombre, que no forman parte de sus características inherentes.

\section{Bibliografía}

Estrada Ramírez, Hortensia, 1996. La lengua sáliba. Clases nominales y sistema de concordancia, Premios Nacionales de Colcultura 1995, Tercer Mundo Editores, Santafé de Bogotá.

\footnotetext{
${ }^{10}$ Nótese que este verbo nominalizado es una base discontinua que infija el morfema de clase nominal.
} 
. 2000. "La lengua sáliba”, en Lenguas indígenas de Colombia: una visión descriptiva, María Stella González de Pérez y María Luisa Rodríguez de Montes (coordinadoras), Instituto Caro y Cuervo, Santafé de Bogotá. . 2001 (enero a junio) "Las marcas de clase nominal y sus funciones en la oración”. Fundación para la Promoción de la Investigación y la Tecnología, Banco de la República, CCELA, Universidad de los Andes, Bogotá.

Data recebimento: 20/03/2014.

Data aceite: 18/04/2014. 